\title{
LncRNA CDKN2B-AS1 promotes the progression of ovarian cancer by miR-143-3p/SMAD3 axis and predicts a poor prognosis
}

\author{
C. XU*, J. ZHAI, Y. FU \\ Department of Gynecology and Obstetrics, Beijing Tongren Hospital Affiliated to Capital Medical University, Beijing, China \\ ${ }^{*}$ Correspondence: gkh6vb@163.com
}

Received June 17, 2019 / Accepted October 8, 2019

\begin{abstract}
Long noncoding RNAs (LncRNAs) show great potential as the therapeutic targets attributing to their implication in the progression of various human cancers, including ovarian cancer $(\mathrm{OC})$. Here, we aimed to explore the biological function of lncRNA cyclin-dependent kinase inhibitor 2B antisense RNA 1 (CDKN2B-AS1) in OC and its mechanism of action. The abundances of CDKN2B-AS1, miR-143-3p, and SMAD3 mRNA were determined by quantitative real-time polymerase chain reaction (qRT-PCR). Cell Counting Kit-8 (CCK8) was performed to analyze cell proliferation. Cell apoptosis was assessed by flow cytometry and western blot analyses. Transwell assay was utilized to analyze cell migration and invasion abilities. Tumor xenograft was performed to confirm the role of CDKN2B-AS1 in ovarian tumor growth in vivo. The protein level of SMAD3 was examined by western blot assay. The interaction between CDKN2B-AS1 and miR-143-3p, or miR-143-3p and SMAD3 was demonstrated by bioinformatic, luciferase reporter, qRT-PCR and western blot analyses. CDKN2B-AS1 was upregulated in $\mathrm{OC}$ and correlated with clinicopathologic features. The knockdown of CDKN2B-AS1 hampered the development of OC, as reflected by the suppression of cell proliferation, migration, and invasion, and the enhancement of cell apoptosis, whereas the effects could be rescued by the overexpression of SMAD3. The absence of CDKN2B-AS1 blocked tumor growth in vivo. CDKN2B-AS1 served as a molecular sponge for miR-143-3p, leading to the derepression of miR-143-3p target SMAD3, which eventually triggered the progression of OC. In conclusion, CDKN2B-AS1 promoted tumor growth, invasion, and migration of OC by regulation of miR-143-3p/SMAD3 axis, hinting that CDKN2B-AS1 might be a potential biomarker for OC diagnosis and treatment.
\end{abstract}

Key words: CDKN2B-AS1, miR-143-3p, SMAD3, ovarian cancer, tumor progression

Ovarian cancer (OC) is the second most common malignant genital tumor in females worldwide, which accounts for almost $2.5 \%$ of all cancer types among females, and $4.9 \%$ of female cancer-related deaths [1]. Despite great advances have been achieved in cytoreduction surgery and adjuvant chemotherapy, OC is still an obstinate gynecological carcinoma with low overall survival rates, largely triggered by the delay of diagnoses [2]. Thereby, a better understanding of the molecular mechanisms associated with carcinogenesis is required for the improvement of OC diagnosis and treatment.

Recently, a large number of long noncoding RNAs (lncRNAs) have been verified to be involved in a diverse range of cellular processes, including cell proliferation, differentiation, cycle arrest, apoptosis, invasion, and migration $[3,4]$. Alterations in the expression of lncRNAs should be responsible for tumorigenesis via regulating a variety of biomolecules at epigenetic, transcriptional, post-transcriptional levels [5]. Furthermore, accumulating evidence and efforts indicate that plenty of lncRNAs play vital roles in the occurrence and development of OC. For instance, a recent study identified a six-lncRNA signature associated with the recurrence of OC patients [6]. Moreover, the upregulation of lncRNA NEAT1, HOTAIR, and CCAT2 contributed to the malignant processes of $\mathrm{OC}$ and they were correlated with the poor prognosis of patients [7-9]. However, lncRNA RP11-190D6.2 played a tumor-suppressive role in OC [10].

Cyclin-dependent kinase inhibitor 2B antisense RNA 1 (CDKN2B-AS1), also known as ANRIL, is a cancer-related lncRNA associated with the etiology of various malignancies. Elevated expression of ANRIL was observed in gastric cancer [11], lung cancer [12], and bladder cancer [13], and exhibited a significant correlation with tumor growth. In addition, a previous study also demonstrated that ANRIL was markedly upregulated in advanced OC, and had the ability to promote cell invasion and tumor metastasis [14]. Moreover, ANRIL obviously triggered cell proliferation and inhibited apoptosis 
in epithelial OC [15]. However, the specific mechanism of CDKN2B-AS1 in OC development and progression remains to be fully elucidated.

In the past decade, lots of pieces of evidence have demonstrated that lncRNAs are regarded as the primary section of the competing endogenous RNA (ceRNA) network, thereby leading to the suppression of miRNA and the derepression of miRNA target mRNA [16]. In our study, we performed a series of experiments to investigate the roles and mechanisms of CDKN2B-AS1 in OC. Results showed that CDKN2B-AS1 was upregulated in $\mathrm{OC}$ and associated with a poor prognosis. Moreover, we confirmed that CDKN2B-AS1 could regulate cell growth, migration, and invasion through upregulation of SMAD3 by silencing miR-143-3p. Our results suggest that CDKN2B-AS1 may serve as a biomarker to facilitate the development of lncRNA-directed diagnostics and therapeutics of OC.

\section{Patients and methods}

Tissue specimens and clinicopathological data. Eightytwo fresh tumor tissues and corresponding noncancerous tissues were obtained from OC patients who underwent surgical resection in Beijing Tongren Hospital Affiliated to Capital Medical University. None of them received any form of treatment before tissue collection. These samples were immediately snap-frozen in liquid nitrogen and store at $-80^{\circ} \mathrm{C}$ for the subsequent study.

According to the median value of CDKN2B-AS1 expression in tumor tissues, OC patients were divided into a high CDKN2B-AS1 group (41 cases) and a low CDKN2B-AS1 group (41 cases). Clinicopathological data in each group, including age, menopause status, histological type, FIGO stage, tumor size, and lymph node metastasis, were obtained from medical records and pathology reports, and are described in Table 1. Then, all patients were followed-up for five years to analyze the correlation between CDKN2B-AS1 expression and survival rates. Those who died due to other diseases rather than $\mathrm{OC}$ were left out of this study. All procedures performed in our study involving human participants were conformed to the standards of the Research Ethics Committee of Beijing Tongren Hospital Affiliated to Capital Medical University. Written informed consent was obtained from all participants prior to this research.

Cell culture. Human ovarian epithelial cells (HOSEpiC) and OC A2780 cell line were purchased from the American Type Culture Collection (ATCC, Manassas, VA, USA). OC cell lines SKOV3, OVCAR3, and HO-8910 were obtained from the Cell Bank of the Chinese Academy of Sciences (Beijing, China). HOSEpiC, A2780, OVCAR3, and HO-8910 cells were grown in RPMI-1640 medium (Gibco, Carlsbad, CA, USA). SKOV3 cells were cultured in specific McCoy's 5A medium (Sigma-Aldrich, St. Louis, MO, USA). All mediums were supplemented with $10 \%$ fetal bovine serum (FBS, Gibco).
Plasmids and transfection. CDKN2B-AS1-specific shorthairpin RNA (sh-CDKN2B-AS1) and non-targeting shRNA (NT shRNA) were synthesized by GenePharma (Shanghai, China). CDKN2B-AS1-overexpressed plasmid (CDKN2BAS1) was constructed by GenePharma by inserting the full-length sequences of CDKN2B-AS1 into the pcDNA 3.1 vector, with the pcDNA 3.1 vector as a negative control (vector). miR-143-3p mimics and its scrambled control (miR-NC mimics), miR-143-3p inhibitor, and relative control (miR-NC inhibitor) were purchased from Thermo Fisher (Waltham, MA, USA). SKOV3 and HO-8910 cells at the confluence of $80 \%$ were transfected with oligonucleotides or plasmids using Lipofectamine 2000 (Invitrogen, Carlsbad, CA, USA). Forty-eight hours thereafter, cells were harvested for later analyses.

Quantitative real-time polymerase chain reaction (qRT-PCR). Total RNA was extracted from tumor tissues and cells by using TRIzol reagent (Invitrogen), followed by the detection of RNA purity using a spectrophotometer (Thermo Fisher). Purified RNA was reverse-transcribed into complementary DNA (cDNA) using High Capacity cDNA Reverse Transcription Kit (Thermo Fisher) or TaqMan ${ }^{\mathrm{TM}}$ Advance miRNA cDNA Synthesis Kit (Thermo Fisher). After that, qRT-PCR was performed using SYBR GreenER ${ }^{\mathrm{TM}} \mathrm{qPCR}$ SuperMix Universal (Thermo Fisher) mixed with special primers and ran on an ABI 7500 Real-time PCR Systems (Applied Biosystems, Foster City, CA, USA). All samples were repeated for three times, and $2^{-\Delta \Delta C t}$ method was used to calculate the relative expression levels by normalizing to $\beta$-actin or U6. Primer sequences are displayed below. CDKN2B-AS1 forward: 5'-TCATCATCATCATCATCATC-3', reverse: 5'-TGCTTCTGTCTCTTCATA-3'. $\beta$-actin forward: 5'-GGCATCGTGATGGACTCCG-3', reverse: 5'-GCTGGAAGGTGGACAGCGA-3'. miR-143-3p RT: 5'-CTCAACTGGTGTCGTGGAGTCGGCAATTCAGTTGAGGAGCTACA-3', forward: 5'-ACACTCCAGCTGGGGTGAGATGAAGCACTG-3', reverse: 5'-TGGTGTCGTGGAGTCG-3'. U6 forward: 5'-CTCGCTTCGGCAGCACA-3', reverse: 5'-AACGCTTCACGAATTTGCGT-3'.

Cell Counting Kit-8 (CCK-8). Cell proliferation was analyzed by using CCK-8 kit (Dojing, Tokyo, Japan) referring to the manufacturer's instructions. In brief, transfected SKOV 3 and HO- 8910 cells $\left(5 \times 10^{3}\right)$ were seeded into 96-well plate. At different time points after transfection $(24 \mathrm{~h}, 48 \mathrm{~h}$, and $72 \mathrm{~h}$ ), $10 \mu \mathrm{l} \mathrm{CCK-8} \mathrm{reagent} \mathrm{was} \mathrm{added} \mathrm{into} \mathrm{each} \mathrm{well} \mathrm{and}$ incubated at $37^{\circ} \mathrm{C}$ for another $2 \mathrm{~h}$. Then, the absorbance at $450 \mathrm{~nm}$ was measured using a microplate reader (Thermo Fisher).

Flow cytometry. Cell apoptosis was assessed using Annexin V-FITC/PI kit (Solarbio, Beijing, China). Transfected cells were digested with EDTA-absented trypsin, washed with PBS and resuspended in binding buffer. Afterward, equal amounts of Annexin V-FITC and propidium iodide (PI) were added and apoptotic cells were observed by flow cytometry (BD Biosciences, Franklin Lakes, NJ, USA). 
Western blot. Total proteins were isolated from SKOV 3 and HO-8910 cells using RIPA lysis buffer (Solarbio). After detection of protein concentration by Pierce BCA Protein Assay Kit (Thermo Fisher), $1 \mu \mathrm{g}$ of protein samples were divided by SDS-PAGE gel and then transferred onto polyvinylidene difluoride membranes (PVDF, Millipore, Bedford, MA, USA). Then, the membranes were blocked with 5\% skimmed milk powder in TBST buffer and probed with primary antibodies against Bcl-2 (1:1000; ab32124; Abcam, Cambridge, USA), Bax (1:1000; ab32503; Abcam), cleaved-caspase 3 (1:1000; ab2302; Abcam), SMAD3 (1:1000; ab40854 Abcam), and GAPDH (1:5000; ab181602; Abcam) overnight at $4^{\circ} \mathrm{C}$. After being washed with TBST buffer for three times, the members were incubated with HRP-labeled second antibody (1:5000; ab205718; Abcam) for $1.5 \mathrm{~h}$ at $37^{\circ} \mathrm{C}$. The protein blots were visualized using an enhanced chemiluminescence kit (ECL, Beyotime, Shanghai, China) and results were quantified using Image Lab software (Bio-Rad, Hercules, CA, USA).

Transwell assays. Cell migration and invasion abilities were evaluated using 24-well Transwell Migration Chambers and Biocoat Matrigel Invasion Chambers (BD Biosciences). SKOV3 and $\mathrm{HO}-8910$ cells $\left(5 \times 10^{4}\right)$ resuspended with serum-free RPMI-1640 medium were added on the upper chambers, and the lower part of the chamber was filled with complete medium containing 10\% FBS. About $16 \mathrm{~h}$ after incubation, cells migrated or invaded onto the basal side of the membrane were fixed with ethanol and stained with crystal violet (Sigma-Aldrich). The migration and invasion cells from five random visual fields were counted using a microscope (Olympus, Tokyo, Japan).

Tumor xenograft model. BALB/c nude mice (aged 4-6 weeks, male) were purchased from Charles River (Beijing, China) and fed under the pathogen-free conditions for one week. SKOV3 cells were infected with $10 \mu \mathrm{l}$ of lentivirus containing the NT shRNA or sh-CDKN2B-AS1. One $\mu \mathrm{g} / \mathrm{ml}$ puromycin (Sigma-Aldrich) was used to screen the infected cells. Then the stably transfected cells were subcutaneously inoculated into the flank of BALB/c nude mice. About 9 days after inoculation, tumor volume was measured every $3^{\text {rd }}$ day according to the volume formula of (length $\times$ width $\left.^{2}\right) / 2$. Next, mice were sacrificed at day 30 after injection and tumors were excised for weight and the qRT-PCR analysis was conducted. The animal experiments performed in our study were approved by the Animal Research Committee of Beijing Tongren Hospital Affiliated to Capital Medical University and all procedures were carried out in line with the Guidelines of Animal Care and Use.

Luciferase reporter assay. Bioinformatics analyses have validated the putative binding sites between CDKN2B-AS1 and miR-143-3p or miR-143-3p and SMAD3 using LncBase Predicted v.2 and Targetscan online software. For luciferase reporter assay, partial sequences of CDKN2B-AS1 and SMAD3 containing putative miR-143-3p binding sites were amplified and cloned into the psiCHECK-2 vector (Promega, Madison, WI, USA) to generate wild-type CDKN2B-AS1 (CDKN2B-AS1 WT) or SMAD3 (SMAD3 WT). Next, the KOD-plus-mutagenesis kit (Toyobo, Osaka, Japan) was utilized to mutate the potential miR-143-3p binding sequences in CDKN2B-AS1 and SMAD3 3'-untranslated region (3'UTR), named CDKN2B-AS1 MUT and SMAD3 MUT. The luciferase reporter was transfected into SKOV3 and HO-8910 cells together with miR-NC mimics or miR-143-3p mimics. At $48 \mathrm{~h}$ thereafter, the luciferase activity was determined using the Pierce Renilla-Firefly Luciferase Dual Assay Kit (Thermo Fisher).

Statistical analysis. All data were displayed as the mean \pm standard deviation (SD) and analyzed using SPSS 20.0 software (SPSS, Inc., Chicago, IL, USA). The differences between groups were assessed by Student's t-test or ANOVA. A p-value $<0.05$ was considered as the statistical difference.

\section{Results}

CDKN2B-AS1 was upregulated in OC and correlated with clinicopathological features. We observed that CDKN2B-AS1 expression was significantly upregulated in ovarian tumor tissues compared with nearby normal tissues, which was detected by qRT-PCR (Figure 1A). Likewise,
A

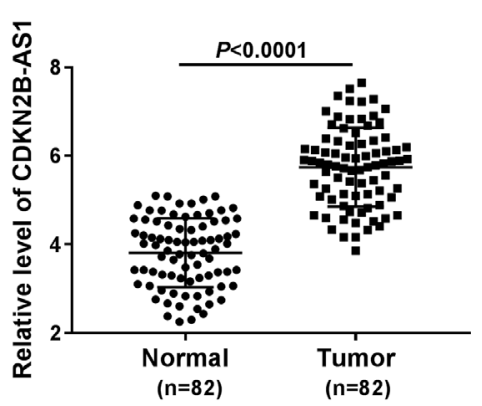

B

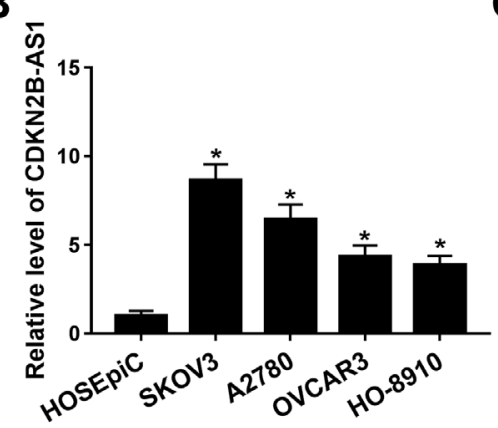

C

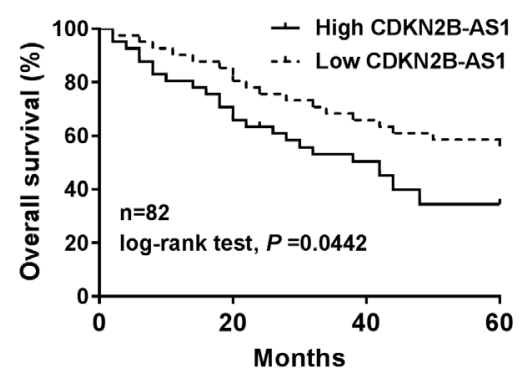

Figure 1. CDKN2B-AS1 was upregulated in OC and associated with poor overall survival. A) The expression of CDKN2B-AS1 in OC tissues and nearby non-tumor tissues was examined by qRT-PCR. B) The expression of CDKN2B-AS1 in a normal ovarian epithelial cell line (HOSEpiC) and OC cell lines (SKOV3, A2780, OVCAR3, and HO-8910) was determined by qRT-PCR. C) Overall survival of OC patients with high or low CDKN2B-AS1 expression was evaluated by Kaplan-Meier survival analysis. ${ }^{*} \mathrm{p}<0.05$ 
elevated abundances of CDKN2B-AS1 were also exhibited in OC cell lines (SKOV3, A2780, OVCAR3, and HO-8910) compared with that in HOSEpiC cells (Figure 1B). Correlation analysis showed that high CDKN2B-AS1 expression was associated with advanced FIGO stage, tumor size, and lymph node metastasis (Table 1). However, there were no significant correlations between CDKN2B-AS1 expression and other parameters such as age, menopause status, ovarian involvement, and histological type (Table 1). Moreover, the KaplanMeier analysis showed that high CDKN2B-AS1 was negatively correlated with poor overall survival of OC patients (Figure 1C). Thereby, these data suggested that CDKN2B-AS1 might function as a major regulator in the progression of OC.

CDKN2B-AS1 promoted cell proliferation, migration, and invasion, while inhibited apoptosis in OC. To explore the roles of CDKN2B-AS1 in OC progression, loss- and gainof-function experiments were performed by interfering or overexpressing CDKN2B-AS1 in SKOV3 or HO-8910 cells. The qRT-PCR assay showed that CDKN2B-AS1 was obviously downregulated in sh-CDKN2B-AS1-transfected SKOV3 cells, and upregulated in CDKN2B-AS1-transfected HO-8910 cells compared to relative controls (Figures 2A, 2B), hinting that sh-CDKN2B-AS1 and CDKN2B-AS1 could be used for the following functional assays. The CCK- 8 assay showed that CDKN2B-AS1 knockdown inhibited, while upregulation promoted cell proliferation in SKOV3 or HO8910 cells (Figures 2C, 2D). Flow cytometric analysis revealed that sh-CDKN2B-AS1 significantly contributed to the apoptosis of SKOV 3 cells, while CDKN2B-AS1 overexpressing plasmid inhibited HO-8910 cell apoptosis (Figure 2E). Moreover, depletion of CDKN2B-AS1 led to a lowered expression of Bcl-2 protein, and elevated expression of Bax and cleaved caspase 3 proteins in SKOV3 cells (Figure 2F). However, CDKN2B-AS1 overexpression in HO-8910 cells showed negative effects. These results further validated the regulatory effect of this lncRNA on cell apoptosis. Transwell assay showed that CDKN2B-AS1 knockdown protected SKOV3 cells from migration and invasion. However, overexpression of CDKN2B-AS1 exhibited an opposite effect on cell migration and invasion in HO-8910 cells (Figures 2G, 2H). Taken together, these data indicated that CDKN2B-AS1 functioned as a positive mediator in the malignant progression of OC.

The absence of CDKN2B-AS1 blocked tumor growth in vivo. We provided the first study to explore the impact of sh-CDKN2B-AS1 on ovarian tumor growth in vivo. The result showed that tumor volume kept increasing after inoculating SKOV3 cells into mice. However, the volume was smaller in the sh-CDKN2B-AS1 group than that in the NT shRNA group (Figure 3A). Similarly, sh-CDKN2B-AS1 exhibited an inhibitory effect on tumor weight (Figure 3B). The result of the qRT-PCR assay showed that sh-CDKN2B-AS1 resulted in the reduction of CDKN2B-AS1 expression in xenograft relative to the negative control (Figure $3 \mathrm{C}$ ). These findings suggested that CDKN2B-AS1 knockdown suppressed tumor growth in vivo.
Table 1. Correlation between the clinicopathological characteristics and CDKN2B-AS1 expression in ovarian carcinoma.

\begin{tabular}{|c|c|c|c|c|}
\hline \multirow[b]{2}{*}{ Factor } & \multirow[b]{2}{*}{ No. } & \multicolumn{2}{|c|}{ CDKN2B-AS1 n (\%) } & \multirow[b]{2}{*}{ p-value } \\
\hline & & $\begin{array}{l}\text { Low expression } \\
\qquad(\mathrm{n}=41)\end{array}$ & $\begin{array}{l}\text { High expression } \\
\qquad(\mathrm{n}=41)\end{array}$ & \\
\hline Age & & & & 0.682 \\
\hline$\leq 53$ & 42 & $19(45.2)$ & $23(54.3)$ & \\
\hline$>53$ & 40 & $22(55.0)$ & $18(45.0)$ & \\
\hline Menopause status & & & & 0.480 \\
\hline Premenopausal & 35 & $20(57.1)$ & $15(42.9)$ & \\
\hline Postmenopausal & 47 & $21(44.7)$ & $26(55.3)$ & \\
\hline \multicolumn{5}{|l|}{ Ovarian involvement } \\
\hline Single side & 34 & $19(55.9)$ & $15(44.1)$ & 0.396 \\
\hline Both side & 48 & $22(45.8)$ & $26(54.2)$ & \\
\hline \multicolumn{5}{|l|}{ Histological type } \\
\hline Serous & 55 & $26(47.3)$ & $29(52.7)$ & 0.421 \\
\hline Nonserous & 27 & $15(55.6)$ & $12(44.4)$ & \\
\hline FIGO stage & & & & $0.021^{*}$ \\
\hline $\mathrm{I}+\mathrm{II}$ & 31 & $20(64.5)$ & $11(35.5)$ & \\
\hline $\mathrm{III}+\mathrm{IV}$ & 51 & $19(37.3)$ & $32(62.7)$ & \\
\hline Tumor size & & & & $0.015^{*}$ \\
\hline$\leq 7.5$ & 47 & $30(63.8)$ & $17(36.2)$ & \\
\hline$>7.5$ & 35 & $11(31.4)$ & $24(68.6)$ & \\
\hline \multicolumn{2}{|c|}{ Lymph node metastasis } & & & $0.008^{*}$ \\
\hline No & 46 & $30(65.2)$ & $16(34.8)$ & \\
\hline Yes & 36 & $11(30.5)$ & $15(69.5)$ & \\
\hline
\end{tabular}

${ }^{\star}$ Statistically significant

CDKN2B-AS1 served as a molecular sponge for miR-143-3p. Bioinformatics analyses using LncBase Predicted v.2 software initially proved the existence of putative CDKN2B-AS1 binding sequences in miR-143-3p (Figure 4A). Next, the luciferase reporter assay was performed to explore the true interaction between CDKN2B-AS1 and miR-143-3p. The results showed that forced expression of miR-143-3p obviously decreased the luciferase activity of CDKN2B-AS1 WT reporter rather that CDKN2B-AS1 MUT reporter in SKOV3 and HO-8910 cells (Figures 4B, 4C). Furthermore, we observed that miR-143-3p expression was lowered in OC cell lines SKOV3 and HO-8910 compared to normal control (Figure 4D). sh-CDKN2B-AS1 stimulated miR-143-3p expression in SKOV3 cells, and CDKN2B-AS1 suppression showed an inhibitory effect on miR-143-3p expression in HO-8910 cells (Figure 4E). Introduction of miR-143-3p mimics facilitated, while miR-143-3p inhibitor attenuated miR-143-3p expression in SKOV3 or HO-8910 cells (Figure $4 \mathrm{~F}$ ). The addition of miR-143-3p degraded the abundance of CDKN2B-AS1 in SKOV3 cells, but miR-143-3p 


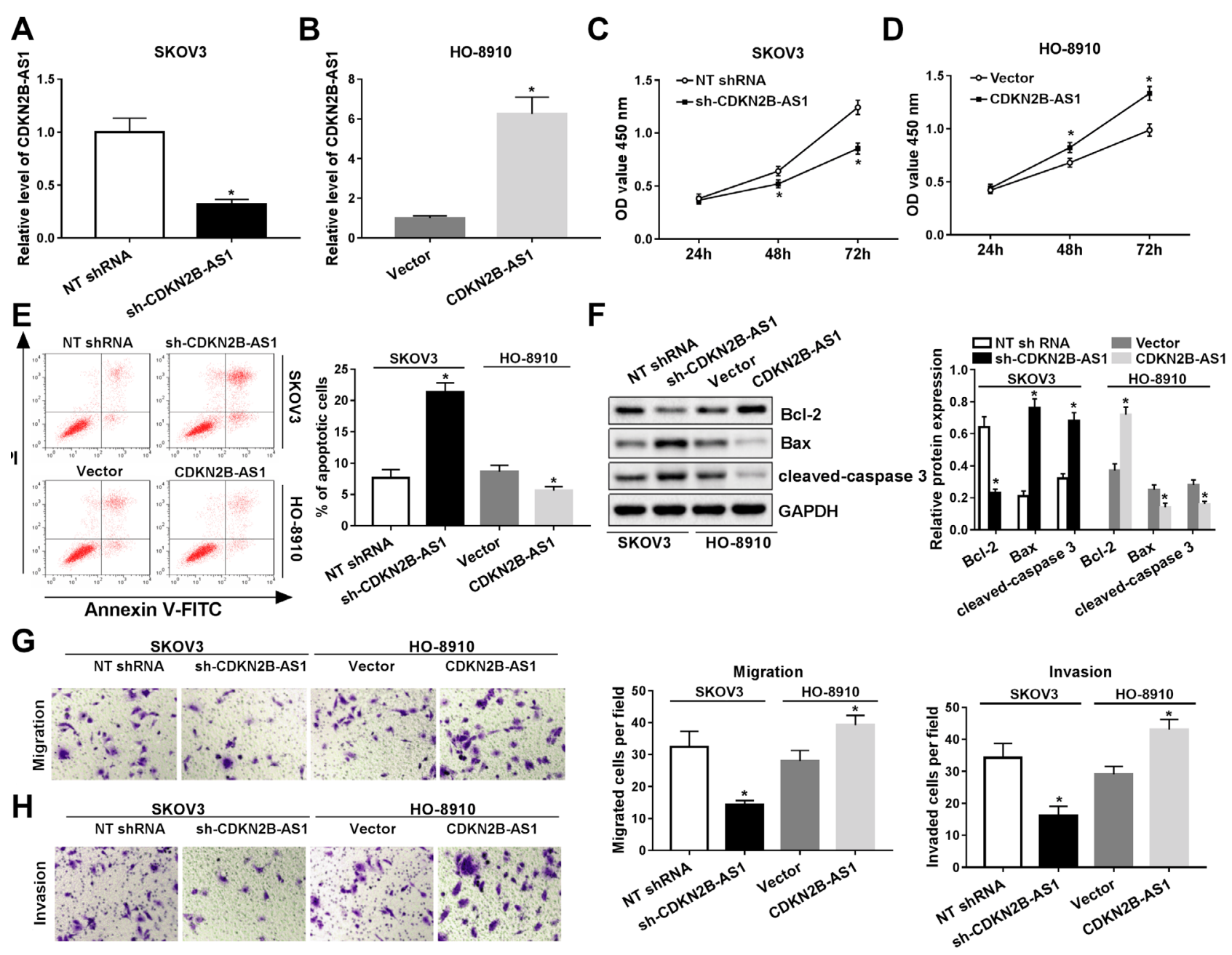

Figure 2. CDKN2B-AS1 promoted OC cell proliferation, migration, and invasion, while inhibited cell apoptosis. SKOV3 cells were transfected with NT shRNA or sh-CDKN2B-AS1, and HO-8910 cells were transfected with Vector, or CDKN2B-AS1. A, B) 48 h thereafter, the abundance of CDKN2BAS1 was detected by $\mathrm{qRT}$-PCR. C, D) CCK-8 assay was performed to determine cell proliferation. E) The cell apoptotic rate was determined by flow cytometry. F) The expression of apoptosis-related proteins Bcl-2, Bax, and cleaved-caspase 3 was examined by western blot assay. G, H) Cell migration and invasion abilities were evaluated by Transwell analysis. ${ }^{*} \mathbf{p}<0.05$

inhibitor showed a negative effect on CDKN2B-AS1 expression in HO-8910 cells (Figure 4G). Next, we also observed the decreased expression of miR-143-3p in ovarian tumor samples compared to counterparts (Figure $4 \mathrm{H}$ ). Correlation analysis showed the negative relationship between CDKN2BAS1 and miR-143-3p expression (Figure 4I). Altogether, our data confirmed that CDKN2B-AS1 functioned as a miR-143-3p sponge in OC.

CDKN2B-AS1 promoted OC progression by sponging miR-143-3p. In the present study, rescue experiments were conducted to probe the regulatory mechanism of CDKN2BAS1 in the malignant progression of ovarian cells. SKOV3 cells were transfected with NT shRNA, sh-CDKN2B-AS1, sh-CDKN2B-AS1+miR-NC inhibitor, or sh-CDKN2BAS1+miR-143-3p inhibitor, and HO-8910 cells were transfected with vector, CDKN2B-AS1, CDKN2B-AS1+miR$\mathrm{NC}$ mimics, or CDKN2B-AS1+miR-143-3p mimics. As the results displayed, the miR-143-3p inhibitor abolished the inhibitory effect of sh-CDKN2B-AS1 on miR-143-3p expression in SKOV3 (Figure 5A). Inversely, miR-143-3p mimics overturned the promotive effect of CDKN2B-AS1 on miR-143-3p expression in HO-8910 cells (Figure 5B). CCK-8 analysis disclosed that knockdown of miR-143-3p abrogated CDKN2B-AS1-inhibited SKOV3 cell proliferation (Figure 5C), while miR-143-3p restoration weakened CDKN2B-AS1-stimulated HO-8910 cell proliferation (Figure 5D). Flow cytometric and western blot analyses showed that the stimulatory impact of sh-CDKN2B-AS1 on SKOV3 cell apoptosis was reversed following miR-143-3p inhibition, which was demonstrated by the changes of apoptotic rate (Figure 5E), and apoptosis-related proteins Bcl-2, Bax, cleaved-caspase 3 (Figures 5G, 5I). The inhibitory impact of CDKN2B-AS1 on HO-8910 cell apoptosis was overturned following miR-143-3p overexpression (Figures 5F, 

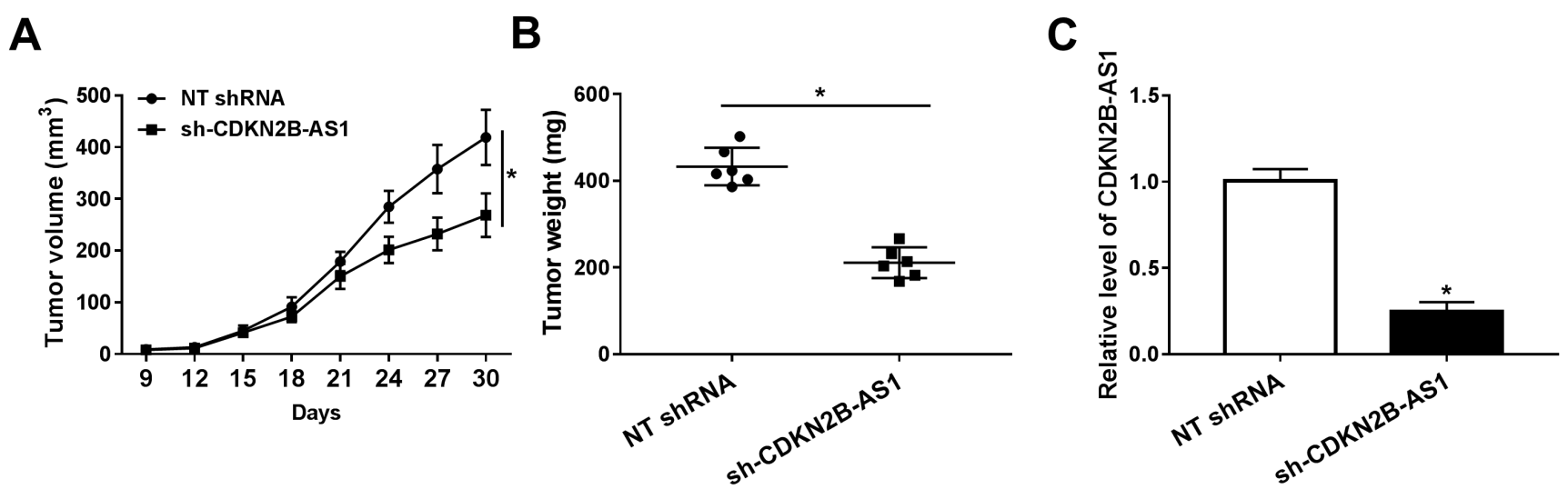

Figure 3. CDKN2B-AS1 knockdown receded ovarian tumor growth in vivo. SKOV3 cells that were stably transfected with NT shRNA or sh-CDKN2BAS1 were subcutaneously injected into mice $(n=6 /$ group). A) Tumor volume at different times after injection was calculated. B) Thirty days after injection, mice were sacrificed and xenografts were harvested for weight. $\mathrm{C}$ ) $\mathrm{CDKN} 2 \mathrm{~B}-\mathrm{AS} 1$ expression in xenografts was measured by $\mathrm{qRT}$-PCR. ${ }^{\star} \mathrm{p}<0.05$
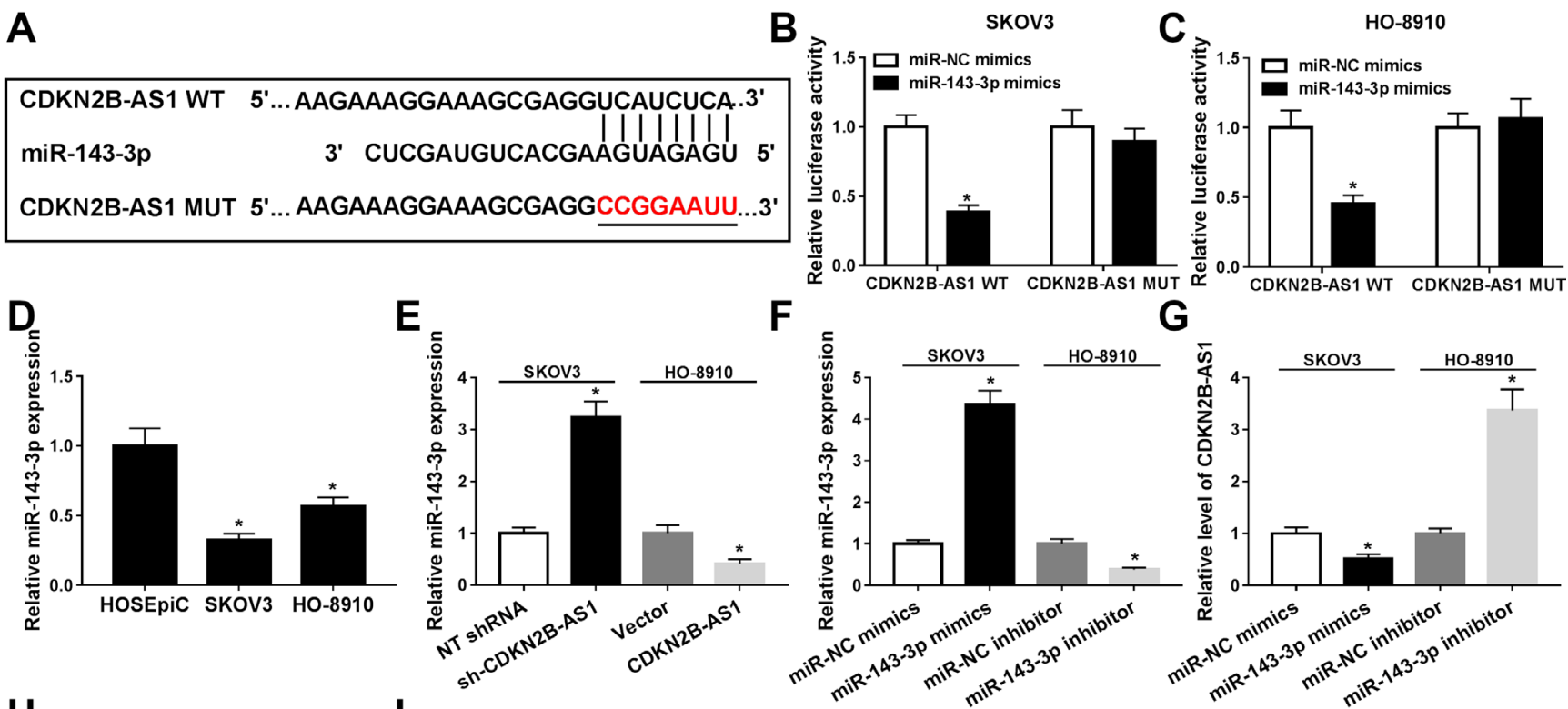

$\mathbf{G}$
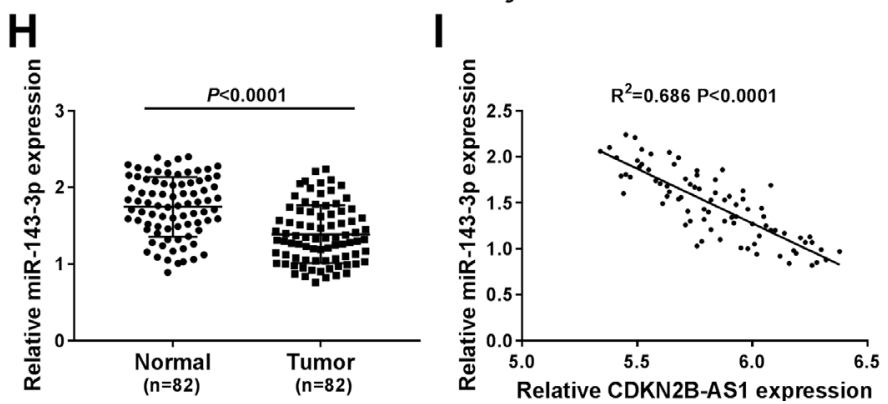

Figure 4. CDKN2B-AS1 served as a miR-143-3p sponge in OC cells. A) The putative binding sequences of CDKN2B-AS1 in miR-143-3p were predicted using the LncBase Predicted v.2 software. B, C) Luciferase activity of wile-type or mutant CDKN2B-AS1 reporter in SKOV3 and HO-8910 cells following the transfection of miR-NC mimics or miR-143-3p mimics was determined by luciferase reporter assay. D) miR-143-3p expression in OC cell lines (SKOV3 and HO-8910) and normal ovarian epithelial cell line (HOSEpiC) was measured by qRT-PCR. E) The expression of miR-143-3p in shCDKN2B-AS1-transfected SKOV3 and CDKN2B-AS1-transfected HO-8910 cells was detected by qRT-PCR. F, G) The expression of miR-143-3p and CDKN2B-AS1 in miR-143-3p mimics-transfected SKOV3 and miR-143-3p inhibitor-transfected HO-8910 cells was detected by qRT-PCR. H) Relative expression of miR-143-3p in OC tumor tissues and adjacent non-tumor tissues was measured by qRT-PCR. I) The relationship between CDKN2B-AS1 and miR-143-3p expression was assessed by correlation analysis. ${ }^{*} \mathrm{p}<0.05$ 


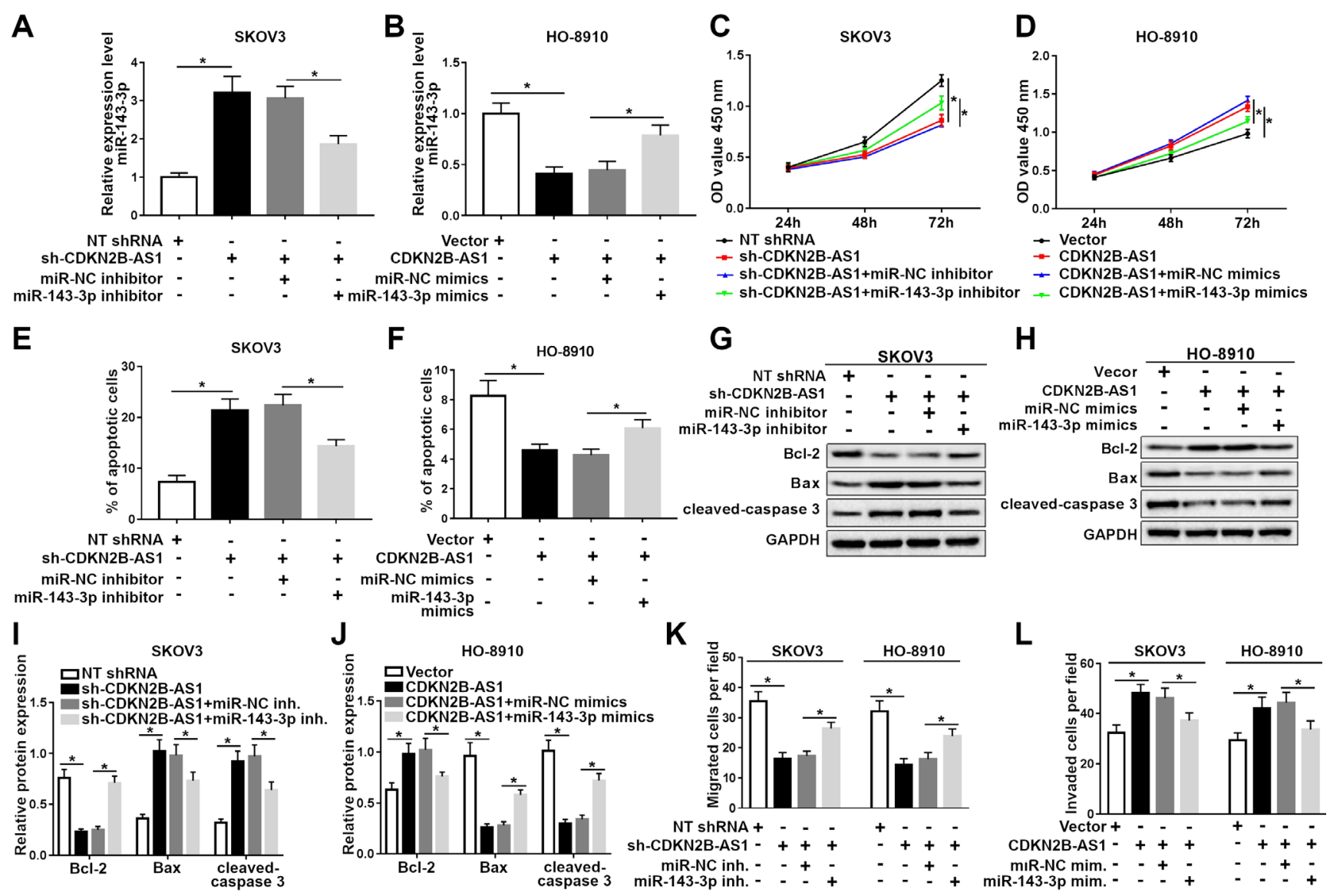

Figure 5. CDKN2B-AS1 promoted the progression of OC cells via sponging miR-143-3p. sh-CDKN2B-AS1 was transfected into SKOV3 cells along with miR-NC inhibitor or miR-143-3p inhibitor. CDKN2B-AS1 was transfected into HO-8910 cells along with miR-NC mimics or miR-143-3p mimics. A, B) The abundance of miR-143-3p was detected by qRT-PCR. C, D) CCK-8 assay was carried out for the determination of cell proliferation. E, F) Cell apoptotic rate was examined by flow cytometry assay. G-J) The expression levels of apoptosis-related proteins Bcl-2, Bax, and cleaved-caspase 3 were measured by western blot assay. $\mathrm{K}, \mathrm{L}$ ) Cell migration and invasion abilities were evaluated by Transwell analysis. ${ }^{\star} \mathrm{p}<0.05 ; \mathrm{mim} .=\mathrm{mimics}, \mathrm{inh}$. = inhibitor

5H, 5J). Transwell analysis revealed that miR-143-3p inhibitor enhanced SKOV3 cell migration and invasion, which were initially suppressed by sh-CDKN2B-AS1 (Figure 5K). Also, miR-143-3p mimics attenuated CDKN2B-AS1-stimulated $\mathrm{HO}-8910$ cell migration and invasion (Figure 5L). In a word, CDKN2B-AS1 contributed to the progression of OC by sponging miR-143-3p.

SMAD3 was an authentic target of miR-143-3p. Bioinformatics analysis was performed to explore the candidate target of miR-143-3p using TargetScan software, and SMAD3 was selected attributing to its implication in various cancer progressions (Figure 6A) [17]. Following luciferase reporter assay further confirmed the true interaction between miR-143-3p and SMAD3, as demonstrated by the decreased luciferase activity of SMAD3 WT reporter in miR-143-3poverexpressed SKOV3 and HO-8910 cells, with no significant change in SMAD3 MUT reporter (Figures 6B, 6C). Next, we observed a lowered expression of SMAD3 in SKOV3 and
HO-8910 cells after transfection of miR-143-3p mimics (Figure 6D), and elevated expression of SMAD3 after transfection of miR-143-3p inhibitor (Figure 6E). SMAD3 expression was notably upregulated in OC cells and tissue samples compared with normal controls (Figures 6F, 6G). Correlation analysis showed the negative relationship between miR-143-3p and SMAD3 expression (Figure 6H). These data suggested that SMAD3 could be negatively modulated by miR-143-3p via direct interaction.

CDKN2B-AS1 positively regulated SMAD3 expression via miR-143-3p. Here, rescue experiments were carried out to investigate whether CDKN2B-AS1 functioned as a major mediator of SMAD3 via miR-143-3p. Notably, the silencing of CDKN2B-AS1 significantly suppressed SMAD3 expression, which was subsequently promoted by miR-143-3p inhibition in SKOV3 cells (Figure 7A). Forced expression of CDKN2B-AS1 overtly upregulated SMAD3, while this effect was reversed by miR-143-3p overexpression in HO-8910 cells 
A

\begin{tabular}{|cc|}
\hline SMAD3 WT & 5'...GCCUGUGCUGGAACAUCAUCUCA...3' \\
miR-143-3p & 3' CUCGAUGUCACGAAGUAGAGU \\
SMAD3 MUT & 5'...GCCUGUGCUGGAACACAGGUCUU....3'
\end{tabular}

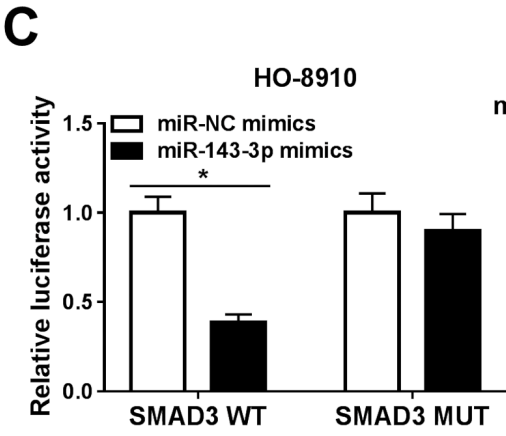

$\mathbf{F}$

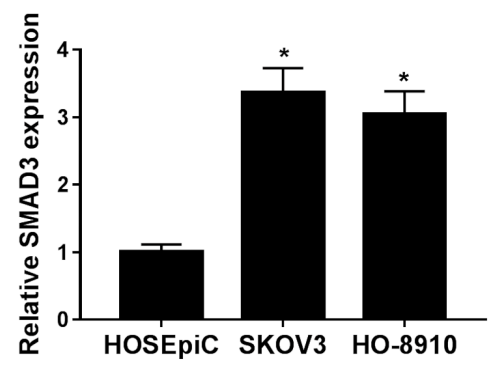

G
B
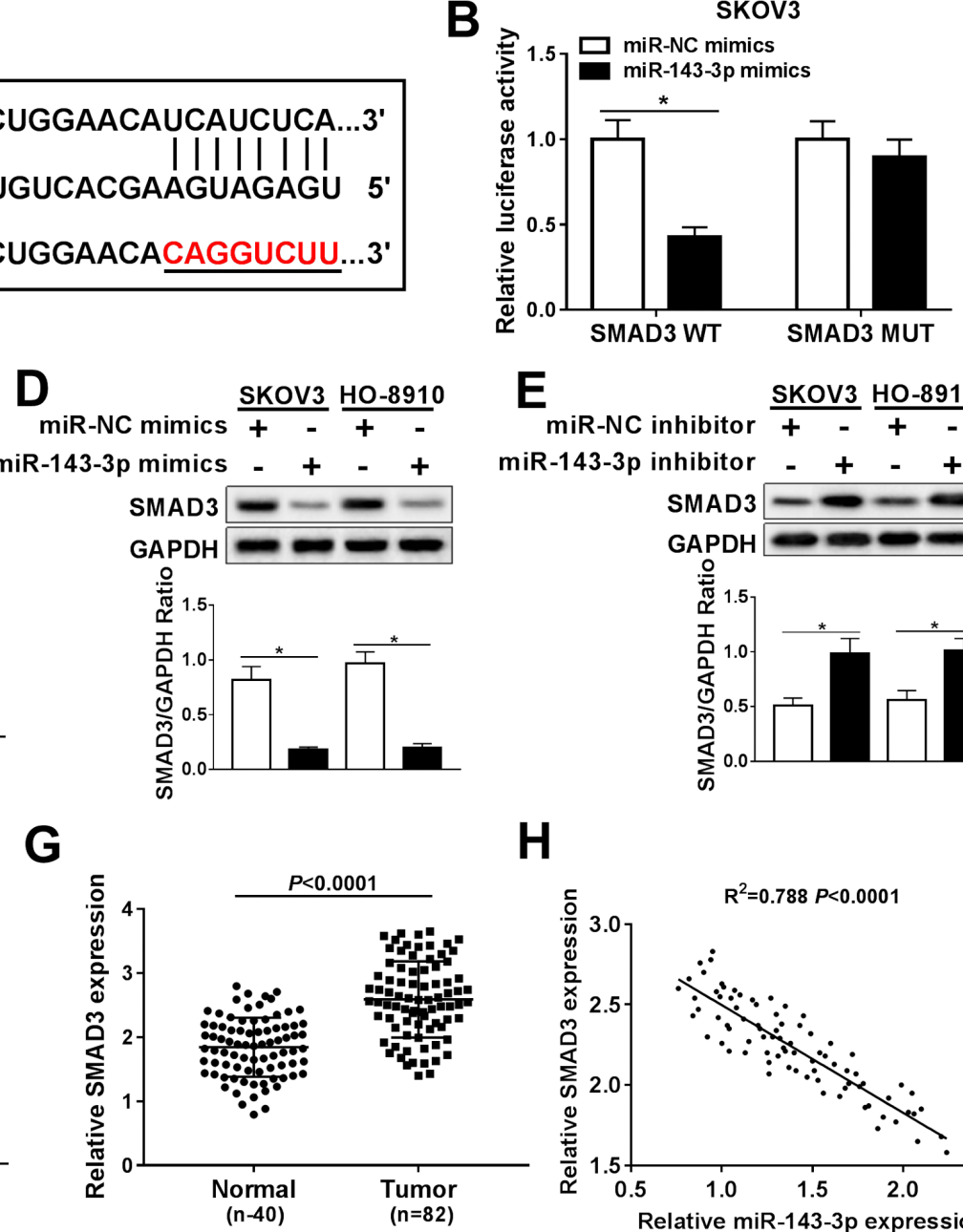

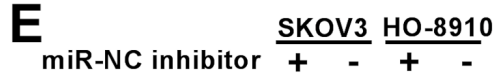

miR-143-3p inhibitor $-+\ldots+$

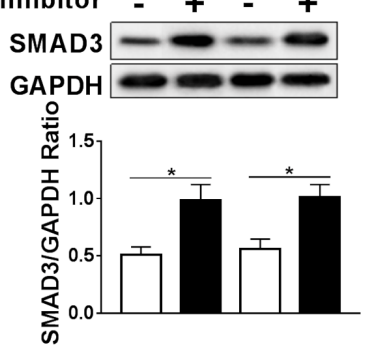

H

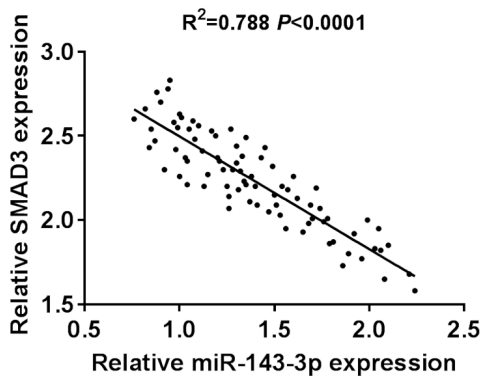

Figure 6. SMAD3 was targeted by miR-143-3p. A) The putative binding regions of miR-143-3p within the in the 3'UTR of SMAD3 were predicted using TargetScan software. B, C) Luciferase activity of SMAD3 WT or SMAD3 MUT reporter in miR-NC mimics or miR-143-3p mimics-transfected SKOV3 and HO-8910 cells were determined by luciferase reporter assay. D, E) The protein levels of SMAD3 in miR-143-3p inhibitor or miR-143-3p mimicstransfected SKOV3 and HO-8910 cells were measured by western blot analysis. F, G) miR-143-3p expression in OC cells and tissues was examined by qRT-PCR. H) The relationship between miR-143-3p and SMAD3 expression in OC tissues was assessed by correlation analysis. ${ }^{*} \mathrm{p}<0.05$

(Figure 7B). Furthermore, a positive relationship between CDKN2B-AS1 and SMAD3 expression in OC tissue samples was observed by correlation analysis (Figure 7C). These results showed that CDKN2B-AS1 resulted in the derepression of SMAD3 via sponging miR-143-3p.

Overexpression of SMAD3 attenuated the impact of CDKN2B-AS1 silencing-mediated inhibition on the progression of $\mathrm{OC}$ cells. To further investigate the roles of CDKN2B-AS1 and SMAD3 in the progression of OC, the expression of SMAD3 in SKOV3 and HO-8910 cells transfected with sh-CDKN2B-AS1 or sh-CDKN2BAS1+SMAD3, as well as their matched controls, was detected by qRT-PCR. The results showed that the level of SMAD3 was downregulated in the sh-CDKN2B-AS1 group, while the expression of SMAD3 was upregulated in the sh-CDKN2BAS1+SMAD3 group (Figure 8A). MTT assay indicated the upregulation of SMAD3 rescued the effect of CDKN2B-AS1 silencing-mediated inhibition on the proliferation of OC cells (Figures 8B, 8C). Moreover, the impact of CDKN2BAS1 silencing-mediated promotion on apoptosis was abated by the overexpression of SMAD3 (Figure $8 \mathrm{D}$ ). At the same time, the downregulation of CDKN2B-AS1 reduced the protein levels of $\mathrm{SMO}$ and $\mathrm{Bcl}-2$ and elevated the levels of Bax and cleaved-caspase 3, whereas SMAD3 overexpression weakened the effects (Figures 8E-H). Further studies elucidated that overexpression of SMAD3 mitigated the impacts of CDKN2B-AS1 silencing-mediated suppression on migration and invasion of OC cells (Figures 8I, 8J). All in all, these results demonstrated that overexpression of SMAD3 rescued the effects of CDKN2B-AS1 silencing-mediated repression on proliferation, migration, invasion, and promotion on apoptosis of OC cells. 
A
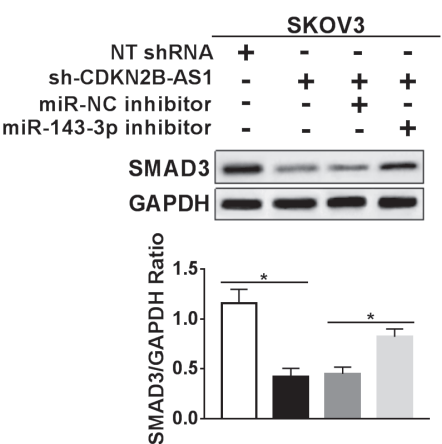
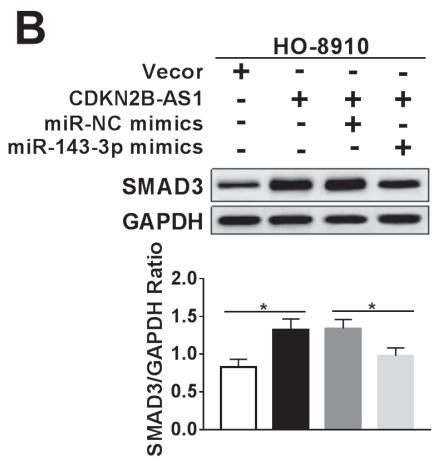

C

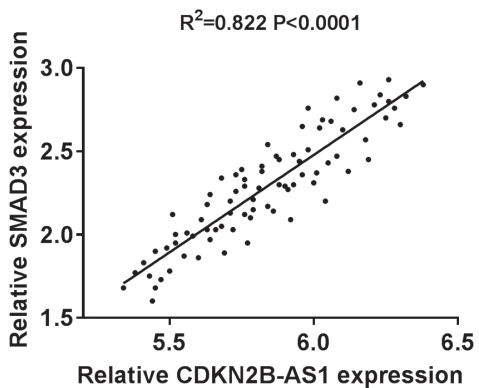

Figure 7. The knockdown of CDKN2B-AS1 resulted in the repression of SMAD3 via sponging miR-143-3p. A) Relative protein expression of SMAD3 was detected by western blot analysis in SKOV3 cells after transfecting with NT shRNA or sh-CDKN2B-AS1, or co-transfecting with sh-CDKN2B-AS1 and miR-NC inhibitor or miR-143-3p inhibitor. B) SMAD3 expression at the protein level was determined by western blot analysis in HO-8910 cells after transfecting with Vector or CDKN2B-AS1, or co-transfecting with CDKN2B-AS1 and miR-NC mimics or miR-143-3p mimics. C) The relationship between CDKN2B-AS1 and SMAD3 expression in OC tissues was assessed by correlation analysis. ${ }^{*} \mathrm{p}<0.05$

\section{Discussion}

To date, large improvements have been achieved to contribute to the prognosis and therapeutics of OC patients. However, OC remains one of the lethal cancers in females due to the late-stage diagnosis. Replacement of lncRNAs presents a novel opportunity for targeted therapy of OC [15]. In the present study, we investigated the role of CDKN2BAS1 in the prognosis and progression of OC and proposed the first integrative view that CDKN2B-AS1 contributed to cell growth, migration, and invasion by modulation of miR-143-3p/SMAD3 axis.

LncRNAs are a type of transcripts larger than 200 nucleotides with limited or no protein-coding capacity. Recently, numerous pieces of evidence disclosed the importance of misregulated lncRNAs in disease pathology, which indicated that these special lncRNAs might provide new insights into the biology of human diseases. Moreover, lncRNAs have emerged as gene regulators and prognostic markers in several malignancies, including OC. For example, human ovarian cancer-specific transcript 2 (HOST2) promoted malignant cell behavior in OC by regulating the availability of let-7b [18]. Nuclear paraspeckle assembly transcript 1 (NEAT1), collaboratively controlled by HuR and miR-124-3p, contributed to OC tumorigenesis and exhibited a significant correlation with the advanced FIGO stage and lymph node metastasis [19]. CDKN2B-AS1 is reported to participate in the regulation of cell phenotypes in various cancers. For instance, CDKN2B-AS1 significantly promoted the proliferation of gastric cancer through a mechanism involving miR-99a/miR-449a [11]. Moreover, CDKN2B-AS1 triggered cell metastasis in lung and thyroid cancer $[20,21]$. Consistent with the previous studies, we found that CDKN2B-AS1 was upregulated in OC patients and cell lines, and its expression was associated with the FIGO stage, tumor size, and lymph node metastasis. The knockdown of CDKN2B-AS1 resulted in the inhibition of cell growth, invasion, and migration. Furthermore, the tumor-suppressive role of CDKN2B-AS1 was also confirmed in vivo. These findings are similar to the study reported by Qiu et al. showing that CDKN2B-AS1 was important in controlling ovarian carcinogenesis [14].

The molecular mechanism by which CDKN2B-AS1 alters cancer-cell phenotype varies by cancer type. Previous studies have validated that CDKN2B-AS1 may serve as ceRNA to sponge miRNA, thereby derepressing miRNA target and influencing cancer-tumor development. Here, we confirmed that CDKN2B-AS1 was an endogenous sponge for miR-143-3p. Moreover, we observed the negative regulation between CDKN2B-AS1 and miR-143-3p expression. miR-143-3p is regarded as an anti-tumor miRNA involved in the regulation of multiple cell phenotypes in several types of cancers, including breast, esophagus, and gastric [22-24]. Furthermore, the tumor-suppressive role of miR-143-3p in ovarian cancer was also demonstrated by Zhang et al., who described that miR-143-3p was notably downregulated in OC and stimulated cell growth via targeting RALBP1 [25]. In the present study, we revealed that restoration of miR-143-3p suppressed CDKN2B-AS1-triggered tumor growth in OC, indicating that CDKN2B-AS1 promoted tumorigenesis by serving as a molecular sponge for miR-143-3p.

Cell growth mediated by transforming growth factorbeta (TGF- $\beta$ ) is a common event during tumorigenesis and metastasis [26]. SMAD3 is a member of the SMAD3 family which functions as a direct mediator of TGF- $\beta$ signaling through forming a complex with SMAD4 and then shuttle to the nucleus [27]. Alteration of the TGF- $\beta$ / SMAD3 signal is associated with the growth, migration, and invasion of different types of cancers, like breast cancer and glioma [28, 29]. Zhao et al. found that SMAD3 could be regulated by TCF and JUN through interactions with histone deacetylases and acetyltransferases [30]. Also, higher SMAD3 was linked to the poor overall survival of OC patients, and SMAD3 
A

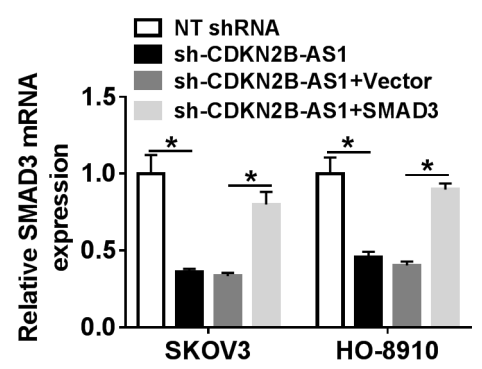

D

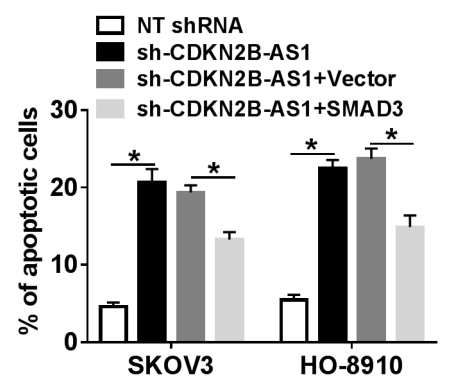

G

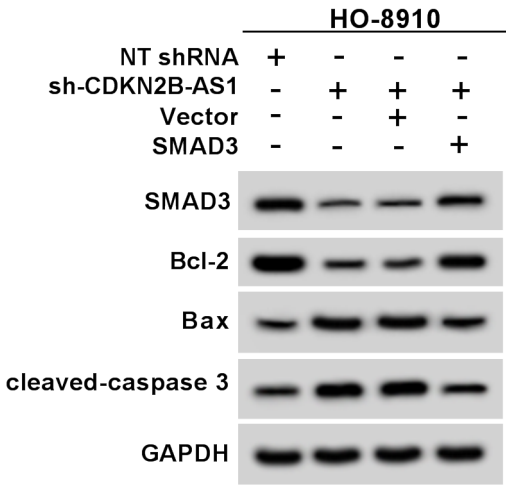

J

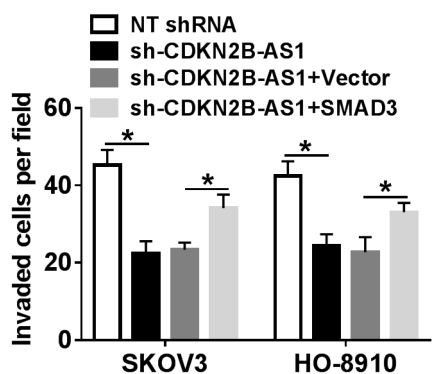

B

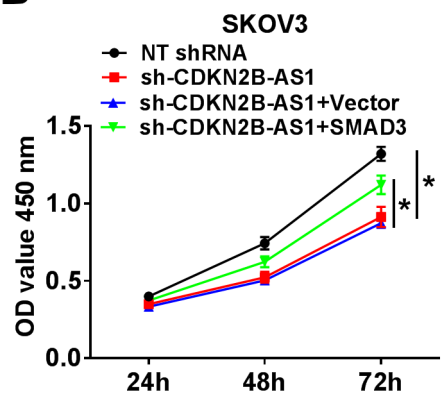

E

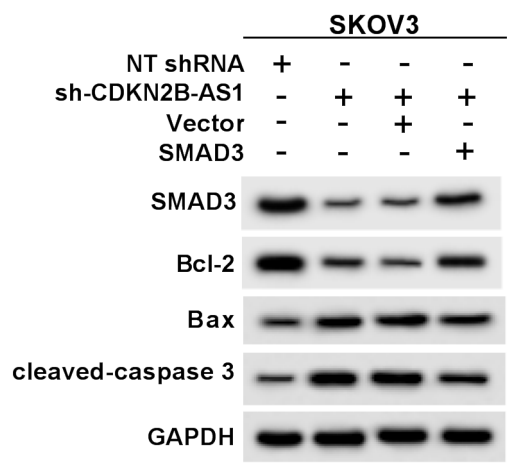

H

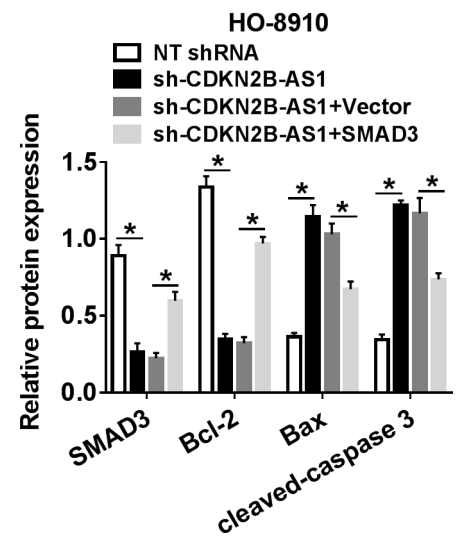

C

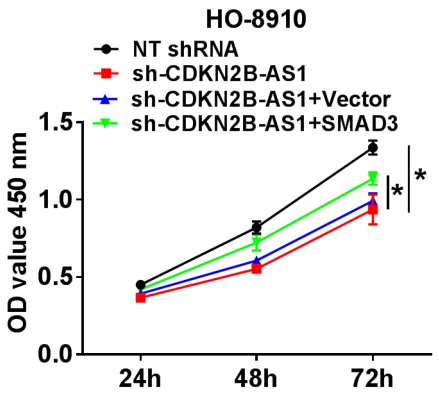

F

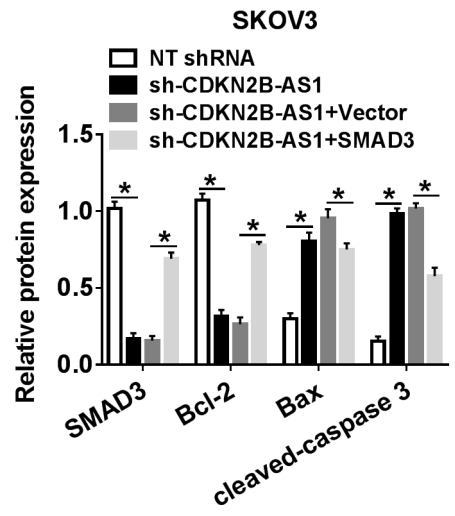

I

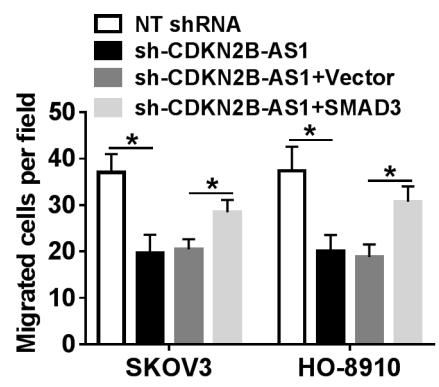

Figure 8. Overexpression of SMAD3 rescued the effects of CDKN2B-AS1 silencing-mediated on proliferation, apoptosis, migration, and invasion. A) The mRNA level of SMAD3 in SKOV3 and HO-8910 cells transfected with sh-CDKN2B-AS1 or sh-CDKN2B-AS1 + SMAD3, as well as the corresponding controls, was detected by qRT-PCR. B, C) CCK-8 assay was conducted to check the proliferation of transfected SKOV3 and HO-8910 cells. D) Flow cytometry was employed to measure the apoptosis of transfected OC cells. E-H) The protein levels of SMAD3, Bcl-2, Bax and cleaved-caspase 3 in transfected OC cells were determined by western blot. I, J) Transwell assay was introduced to assess the abilities of migration and invasion. ${ }^{*} \mathbf{p}<0.05$ 
interference abrogated TGF- $\beta$-induced cell invasion and MMP secretion in ovarian cancer [31]. In view of the implication of SMAD3 in OC progression, we hypothesized that miR-143-3p functioned as a suppressive factor in the development of OC possibly by interacting with SMAD3. Our data showed that miR-143-3p notably decreased the SMAD3 protein level via direct targeting in OC cells. An elevated abundance of SMAD3 in OC tissues had a negative correlation with miR-143-3p. Furthermore, the knockdown of miR-143-3p abolished sh-CDKN2B-AS1-suppressed SMAD3 expression in SKOV3 cells, while restoration of miR-143-3p counteracted the stimulatory effect of CDKN2B-AS1 on SMAD3 expression in HO-8910 cells. Besides, the upregulation of SMAD3 alleviated the effect of CDKN2B-AS1 silencing-mediated suppression on the progression of OC.

In conclusion, CDKN2B-AS1 regulates cell proliferation, apoptosis, invasion, and migration through the miR-143-3p/ SMAD3 axis. These findings highlight the detailed mechanism of CDKN2B-AS1/miR-143-3p/SMAD3 network in OC progression, indicating that CDKN2B-AS1 may be an effective target for the diagnosis and treatment of ovarian cancer.

\section{References}

[1] SIEGEL RL, MILLER KD, JEMAL A. Cancer statistics, 2018. CA Cancer J Clin 2018; 68: 7-30. https://doi.org/10.3322/ caac. 21442

[2] TORRE LA, TRABERT B, DESANTIS CE, MILLER KD, SAMIMI G et al. Ovarian cancer statistics, 2018. CA Cancer J Clin 2018; 68: 284-296. https://doi.org/10.3322/caac.21456

[3] ZHAO L, HAN T, LI Y, SUN J, ZHANG S et al. The lncRNA SNHG5/miR-32 axis regulates gastric cancer cell proliferation and migration by targeting KLF4. FASEB J 2017; 31: 893-903. https://doi.org/10.1096/fj.201600994R

[4] LU MY, LIAO YW, CHEN PY, HSIEH PL, FANG CY et al. Targeting LncRNA HOTAIR suppresses cancer stemness and metastasis in oral carcinomas stem cells through modulation of EMT. Oncotarget 2017; 8: 98542-98552. https://doi. org/10.18632/oncotarget. 21614

[5] ZHU JJ, FU HJ, WU YG, ZHENG XF. Function of lncRNAs and approaches to lncRNA-protein interactions. Sci China Life Sci 2013; 56: 876-885. https://doi.org/10.1007/s11427013-4553-6

[6] YANG K, HOU Y, LI A, LI Z, WANG W et al. Identification of a six-lncRNA signature associated with recurrence of ovarian cancer. Sci Rep 2017; 7: 752. https://doi.org/10.1038/ s41598-017-00763-y

[7] CHEN ZJ, ZHANG Z, XIE BB, ZHANG HY. Clinical significance of up-regulated lncRNA NEAT1 in prognosis of ovarian cancer. Eur Rev Med Pharmacol Sci 2016; 20: 3373-3377.

[8] QIU JJ, WANG Y, DING JX, JIN HY, YANG G et al. The long non-coding RNA HOTAIR promotes the proliferation of serous ovarian cancer cells through the regulation of cell cycle arrest and apoptosis. Exp Cell Res 2015; 333: 238-248. https://doi.org/10.1016/j.yexcr.2015.03.005
[9] HUANG S, CHENG Q, HUANG Z, ZHU Y. The long noncoding RNA CCAT2 is up-regulated in ovarian cancer and associated with poor prognosis. Diagn Pathol 2016; 11: 49. https://doi.org/10.1186/s13000-016-0499-x

[10] TONG W, YANG L, YU Q, YAO J, HE A. A new tumor suppressor lncRNA RP11-190D6.2 inhibits the proliferation, migration, and invasion of epithelial ovarian cancer cells. Onco Targets Ther 2017; 10: 1227-1235. https://doi. org/10.2147/OTT.S125185

[11] ZHANG EB, KONG R, YIN DD, YOU LH, SUN M et al. Long noncoding RNA ANRIL indicates a poor prognosis of gastric cancer and promotes tumor growth by epigenetically silencing of miR-99a/miR-449a. Oncotarget 2014; 5: 22762292. https://doi.org/10.18632/oncotarget.1902

[12] NIE FQ, SUN M, YANG JS, XIE M, XU TP et al. Long noncoding RNA ANRIL promotes non-small cell lung cancer cell proliferation and inhibits apoptosis by silencing KLF2 and P21 expression. Mol Cancer Ther 2015; 14: 268-277. https://doi.org/10.1158/1535-7163.MCT-14-0492

[13] ZHU H, LI X, SONG Y, ZHANG P, XIAO Y et al. Long non-coding RNA ANRIL is up-regulated in bladder cancer and regulates bladder cancer cell proliferation and apoptosis through the intrinsic pathway. Biochem Biophys Res Commun 2015; 467: 223-228. https://doi.org/10.1016/j. bbrc.2015.10.002

[14] QIU JJ, LIN YY, DING JX, FENG WW, JIN HY et al. Long non-coding RNA ANRIL predicts poor prognosis and promotes invasion/metastasis in serous ovarian cancer. Int J Oncol 2015; 46: 2497-2505. https://doi.org/10.3892/ ijo.2015.2943

[15] QIU JJ, WANG Y, LIU YL, ZHANG Y, DING JX et al. The long non-coding RNA ANRIL promotes proliferation and cell cycle progression and inhibits apoptosis and senescence in epithelial ovarian cancer. Oncotarget 2016; 7: 3247832492. https://doi.org/10.18632/oncotarget.8744

[16] SONG J, YE A, JIANG E, YIN X, CHEN Z et al. Reconstruction and analysis of the aberrant lncRNA-miRNA-mRNA network based on competitive endogenous RNA in CESC. J Cell Biochem 2018; 119: 6665-6673. https://doi.org/10.1002/ jcb. 26850

[17] TANG MK, ZHOU S, MENG XM, WANG QM, LI CJ et al. Smad3 promotes cancer progression by inhibiting E4BP4mediated NK cell development. Nat Commun 2017; 8: 14677. https://doi.org/10.1038/ncomms14677

[18] GAO Y, MENG H, LIU S, HU J, ZHANG Y et al. LncRNAHOST2 regulates cell biological behaviors in epithelial ovarian cancer through a mechanism involving microRNA let-7b. Hum Mol Genet 2015; 24: 841-852. https://doi.org/10.1093/ hmg/ddu502

[19] CHAI Y, LIU J, ZHANG Z, LIU L. HuR-regulated lncRNA NEAT1 stability in tumorigenesis and progression of ovarian cancer. Cancer Med 2016; 5: 1588-1598. https://doi. org/10.1002/cam 4.710

[20] ZHAO JJ, HAO S, WANG LL, HU CY, ZHANG S et al. Long non-coding RNA ANRIL promotes the invasion and metastasis of thyroid cancer cells through TGF- $\beta /$ Smad signaling pathway. Oncotarget 2016; 7: 57903-57918. https://doi. org/10.18632/oncotarget.11087 
[21] LIN L, GU ZT, CHEN WH, CAO KJ. Increased expression of the long non-coding RNA ANRIL promotes lung cancer cell metastasis and correlates with poor prognosis. Diagn Pathol 2015; 10: 14. https://doi.org/10.1186/s13000-015-0247-7

[22] LI D, HU J, SONG H, XU H, WU C et al. miR-143-3p targeting LIM domain kinase 1 suppresses the progression of triple-negative breast cancer cells. Am J Transl Res 2017; 9: 2276-2285.

[23] HE Z, YI J, LIU X, JING C, HAN S et al. MiR-143-3p functions as a tumor suppressor by regulating cell proliferation, invasion and epithelial-mesenchymal transition by targeting QKI-5 in esophageal squamous cell carcinoma. Mol Cancer 2016; 15: 51. https://doi.org/10.1186/s12943-016-0533-3

[24] WU XL, CHENG B, LI PY, HUANG HJ, ZHAO Q et al. MicroRNA-143 suppresses gastric cancer cell growth and induces apoptosis by targeting COX-2. World J Gastroenterol 2013; 19: 7758-7765. https://doi.org/10.3748/wjg.v19. i43.7758

[25] ZHANG H, LI W. Dysregulation of micro-143-3p and BALBP1 contributes to the pathogenesis of the development of ovarian carcinoma. Oncol Rep 2016; 36: 3605-3610. https:// doi.org/10.3892/or.2016.5148

[26] FYNAN TM, REISS M. Resistance to inhibition of cell growth by transforming growth factor-beta and its role in oncogenesis. Crit Rev Oncog 1993; 4: 493-540.
[27] MOUSTAKAS A, HELDIN CH. The regulation of TGF $\beta$ signal transduction. Development 2009; 136: 3699-3714. https://doi.org/10.1242/dev.030338

[28] ZHAO Y, MA J, FAN Y, WANG Z, TIAN R et al. TGF- $\beta$ transactivates EGFR and facilitates breast cancer migration and invasion through canonical Smad3 and ERK/Sp1 signaling pathways. Mol Oncol 2018; 12: 305-321. https://doi. org/10.1002/1878-0261.12162

[29] LI Y, ZHANG X, CHEN D, MA C. Let-7a suppresses glioma cell proliferation and invasion through TGF- $\beta / S \operatorname{Smad} 3$ signaling pathway by targeting HMGA2. Tumour Biol 2016; 37 : 8107-8119. https://doi.org/10.1007/s13277-015-4674-6

[30] ZHAO Q, WIRKA R, NGUYEN T, NAGAO M, CHENG P et al. TCF21 and AP-1 interact through epigenetic modifications to regulate coronary artery disease gene expression. Genome Med 2019; 11: 23. https://doi.org/10.1186/s13073019-0635-9

[31] DO TV, KUBBA LA, DU H, STURGIS CD, WOODRUFF TK. Transforming growth factor-beta1, transforming growth factor-beta2, and transforming growth factor-beta3 enhance ovarian cancer metastatic potential by inducing a Smad3dependent epithelial-to-mesenchymal transition. Mol Cancer Res 2008; 6: 695-705. https://doi.org/10.1158/1541-7786. MCR-07-0294 